\title{
Epilepsy in patients with insulin-dependent diabetes and relation to glutamic acid decarboxylase 65
}

Adel A. Mahmoud, MRCP, FPNC, Tayseer Abdelmagid, BPNA, Mohammed AlGhofely, ABP, Abmed M. Hamed, MBBS, Roaa Al Sharif, BHSC, MSN.

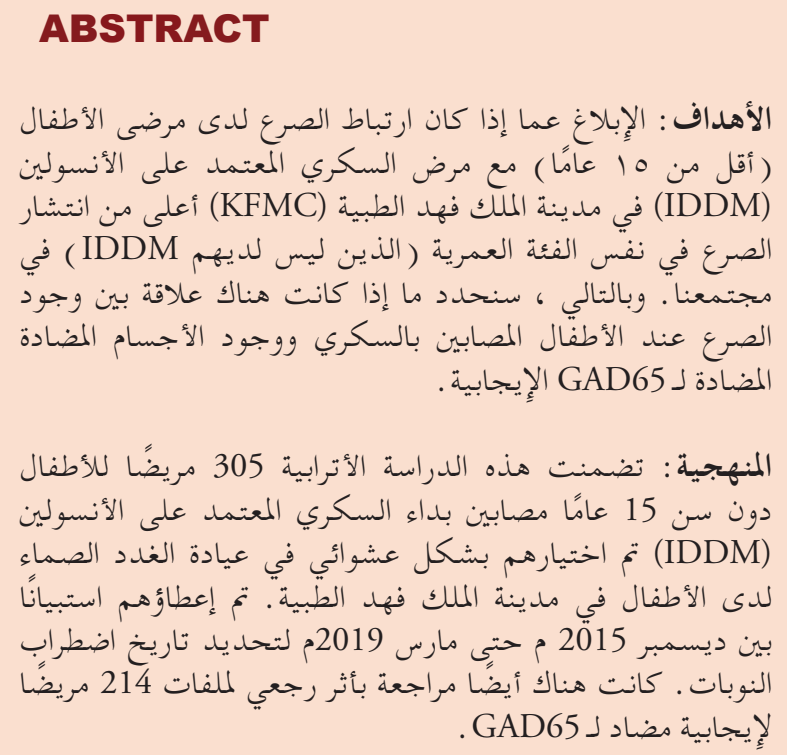

Objectives: To report if the association of epilepsy in pediatric patients (below the age of 15 years) with Insulin-dependent Diabetes (IDDM) at King Fahad Medical City (KFMC) is higher than the prevalence of epilepsy in the same age group (who have no IDDM) in our community. Consequently, we would determine if there is a relationship between the presence of epilepsy in diabetic children and the presence of positive antiGAD65 antibodies.

Methods: This cohort study included 305 pediatric patients below the age of 15 years with Insulindependent Diabetes Mellitus (IDDM). They were randomly recruited at the Pediatric Endocrinology Clinic in KFMC. The patients' caregivers were given a questionnaire between December 2015 till March 2019 to determine the seizure disorder history. There was also a retrospective review of 214 patients' files for anti-GAD 65 positivity.

Results: Our study found a significant relation between the presence of epilepsy in children with IDDM. Therefore, we could confirm the relationship between the existence of epilepsy in children with IDDM and having positive GAD65 antibodies.

Conclusion: Our study supports the presence of consistent relation between having IDDM and having epilepsy in children and between the latter and the presence of positive GAD65 antibodies. Neurosciences 2020; Vol. 25 (3): 200-204
doi: 10.17712/nsj.2020.3.20190057

From the Department of Pediatric Neurology (Mahmoud), National Neuroscience Institute, Department of Pediatric Endocrinology (AlGhofely), Obesity, Endocrine and Metabolism Center, Department of Research (Al Sharif), King Fahad Medical City, Riyadh, and from the Department of Pediatrics (Abdelmagid), Armed Forces Hospital Southern Region, Khamis Mushait, Kingdom of Saudi Arabia,

Received 11th July 2019. Accepted 24th February 2020.

Address correspondence and reprint request to: Dr. Adel A. Mahmoud, Department of Pediatric Neurology, National Neuroscience Institute, King Fahad Medical City, Riyadh, Kingdom of Saudi Arabia. E-mail: amahmoud2000@hotmail.com

ORCID ID: https://orcid.org/0000-0002-1810-839X

$\mathrm{I}$ nsulin dependent diabetes Mellitus (IDDM) is a common condition in children and adolescents worldwide and so is epilepsy. ${ }^{1,2}$ Recently, there were increasing reports suggesting a potential association between having IDDM and the occurrence of epilepsy. ${ }^{3}$ Their association might represent simply a chance to relate their underlying mechanisms. However, the cause- 
effect relationship is not fully well defined. Literature from other countries have shown the increased prevalence of seizure disorders in this group of patients. ${ }^{4,5}$ There are scarce studies in the literature investigating IDDM characteristics contributing to having epilepsy, including positive GAD 65 antibodies. In this study that ran in King Fahad Medical City, Riyadh, Kingdom of Saudi Arabia (KSA) we are aiming to determine if the prevalence of epilepsy among 1DDM children under the age of 15 years (in our center) is higher than controls (same age without IDDM), and to check the positivity of anti-GAD 65 amongst those patients in order to find if there is a relationship between epilepsy in children with diabetes and the presence of positive GAD65 Antibodies.

Methods. This is a retrospective, cross sectional study involving patient survey and chart review. We defined epilepsy as per International League Against Epilepsy (ILAE) "a disorder of the brain characterized by an enduring predisposition to generate epileptic seizures and by the neurobiological, cognitive, psychological, and social consequences of this condition. It requires the occurrence of at least one epileptic seizure" ${ }^{6}$

We randomly recruited 305 pediatric patients having IDDM and below the age of 15 years from our cohort at the Pediatric Endocrinology Clinic, KFMC, KSA. Caregivers were given a questionnaire from December 2015 to March 2019 to determine history of seizures. A retrospective review of 214 files for anti-GAD 65 antibody positivity was carried out as well.

Sample size. A biostatistician determined the sample size based on the prevalence of IDDM in children in Kingdom of Saudi Aabia. A sample size of 256 was sufficient to determine the relationship between epilepsy and the presence of positive anti-GAD antibodies. We investigated 305 children with IDDM and evaluated whether they had epilepsy.

Study setting. The study size was the outpatient's Pediatric Endocrinology Clinic, KFMC. Aquestionnaire was distributed between December 2015 and March 2019.

Data collection. We collected demographic data as well as medical history including family history, perinatal

Disclosure. Authors have no conflict of interests, and the work was not supported or funded by any drug company. This study was funded by King Fahad Medical City, Riyadh, Kingdom of Saudi Arabia (IRF No: 017-021) details, past history, number of hospital admissions, admissions to PICU, number of hypoglycemic attacks, diabetic ketoacidosis or other types of attacks. Subjects also received a comprehensive general, systemic, and neurologic examination. Laboratory investigations included GAD 65 autoantibodies. A $5 \mathrm{ml}$ blood sample was collected from each patients who had not been previously evaluated. A questionnaire was given to the family to evaluate seizure history. Patients summary is shown in Table 1.

Inclusion criteria. We enrolled all IDDM patients younger than 15 years attending the outpatient pediatric endocrinology clinics in KFMC.

Exclusion criteria. We excluded subjects with other risk factors including symptomatic seizures such as brain structural defects, neurodegenerative diseases, other metabolic errors, etc. We excluded subjects whose legal guardians refused to consent.

Off-Study. No patient was considered off the study and none requested to be removed.

End of the study. The targeted duration of the study was 18 months, but many issues led to amendments. The study was finished in March 2019.

Ethical considerations. International standards of Good Clinical Practice (International Conference on Harmonization guidelines), applicable government regulations and institutional research policies and procedures were followed. Our local Institutional Review Board (IRB Log No. 11-125) approved the protocol and its amendments. All subjects or their guardians signed a consent form describing the study. This consent provided sufficient information for subjects to make an informed decision about their participation.

Table 1 - Characteristics of 305 pediatric patients below the age of 15 years with IDDM were randomly recruited at the Pediatric Endocrinology Clinic in KFMC.

\begin{tabular}{lcc}
\hline Characteristics & Mean (SD) & $\mathbf{n}(\%)$ \\
\hline Age (years) & $9.91(3.35)$ & \\
IDDM Onset (years) & $5.82(3.66)$ & \\
Gender (N=204) & \\
Female & $167(54.75)$ \\
Male & $138(45.25)$ \\
Hypoglycemia Seizure (Yes) & $43(14.14)$ \\
Febrile Seizure (Yes) & $4(1.31)$ \\
HX of Epilepsy (Yes) & $13(4.26)$ \\
HX of Febrile Seizure (Yes) & $5(1.64)$ \\
HX of IDDM (Yes) & $118(38.69)$ \\
Anti-GAD (Positive) & $103(33.77)$ \\
\hline \multicolumn{2}{c}{ IDDM - Insulin dependent diabetes Mellitus, HX - history } \\
\hline
\end{tabular}


Statistics. Statistical analysis was carried out in IBM SPSS Statistics 25 software (Ibm.com, 2019). Chi-square $\chi 2$ (2) and independent-samples t test (Statistics.laerd. com, 2019) were used to analyze relationship between groups, with the significance level set at $\alpha=0.05 .7,8$

Results. This cohort $(\mathrm{N}=305)$ included 138 males and 167 females; with average age of 9.82 years and standard deviation of 3.35 , and average IDDM of 4.77 years and standard deviation of 3.41. The rate of seizure disorder was $4.26 \% \quad(\mathrm{n}=13) \quad(p<0.001)$. Anti-GAD 65 antibodies were assayed in $66.89 \%$ of the cohort $(\mathrm{n}=204)$. This was positive in $103(50.49 \% ; \mathrm{n}=103$; $p<0.001$ ).

Results of the chi-square $\chi^{2}$ tests revealed that the rate of positivity was not related to gender, family history of diabetes, or glucose levels.

Discussion. The IDDM is defined by WHO as a metabolic disorder resulting from defects in insulin secretion. It is a common; but non-communicable disease worldwide. It is chronic, progressive and immune-mediated. It may occur in association with other autoimmune diseases. ${ }^{9}$ In most developed countries, IDDM is considered the fifth leading cause of death ${ }^{5}$ IDDM is the most frequent form of diabetes in pediatric populations representing more than $90 \%$ of all cases. ${ }^{10}$

The pathophysiological cause of IDDM is related to the destruction of pancreatic B islet cells. Autoantibodies to islet cell antigens such as GAD65 accompany the T cell-mediated process. GAD catalyzes the conversion of $\mathrm{L}$ glutamic acid to gamma amino butyric acid (GABA) - the major inhibitory neurotransmitter in brain. ${ }^{11}$ The GAD65 is mainly expressed in GABA-ergic neurons and in pancreatic beta cells. ${ }^{12}$

An increasing association between IDDM and epilepsy has been detected in recent years. A UK review evaluated the prevalence of epilepsy in children younger than 16 years with IDDM and found it to be $21 / 1000$, which is greater than the prevalence of epilepsy in the general population. ${ }^{9}$ Epilepsy may be either linked to the primary disease or secondary to the pro-inflammatory processes.

Other studies confirmed an increase in the incidence of IDDM in children below the age of 15 years with a peak age of 10-14 years, ${ }^{5,10,13}$ Seven hundred and five out of 45847 patients with IDDM had epilepsy for a prevalence of 15.5 of 1000 was documented in a large observational cohort study in Germany and Austria from 1995 to 2010. This reflected an increased prevalence of epileptic seizures in diabetic children and adolescents.

The study showed no difference in the GAD autoantibodies between epileptic diabetic patients and non-epileptic diabetic patients. ${ }^{12}$ The same conclusion was found in another retrospective population-based cohort study in Taiwan. This group found that patients below the age of 18 years with IDDM have a significantly higher incidence of epilepsy than patients without IDDM $(p<0.0001) .{ }^{4}$

Keezer et $\mathrm{al}^{5}$ reported that the prevalence of IDDM in epileptics was between 2.3 and $2.9 \%$. Compared to those without epilepsy, the ratio represents a more than two-fold increase in the prevalence of IDDM in those with epilepsy. Another retrospective study in France showed that there is an association between epilepsy and IDDM. ${ }^{14}$ Idiopathic generalized epilepsy and non-idiopathic temporal lobe epilepsy were the most common in this study.

Farsani et $\mathrm{a}^{15}$ studied the incidence of nine common chronic comorbidities with IDDM in children. They found a higher incidence of six chronic diseases including epilepsy in the early years of developing this disease as compared to unaffected children.

Importantly, other studies have shown the opposite results. O'Connell et $\mathrm{al}^{16}$ found no frequency difference between epilepsy in children and adolescents with or without IDDM. Merce et $\mathrm{al}^{17}$ (2014) did not share the hypothesis that epilepsy is more frequent in children with IDDM. While these 2 studies showed contrasting results, the mainstream consensus based on many different international studies is that there is a definite increase in epilepsy prevalence in IDDM-affected children.

Many autoimmune or inflammatory disorders are associated with epilepsy. The hypothesis that some forms of epilepsy with unknown etiology may have an autoimmune basis is gaining a growing support. In many central nervous system (CNS) disorders with recurrent seizures, autoantibodies have been found. ${ }^{18}$ Epilepsy and diabetes mellitus have been encountered in individuals more frequently than might be expected by chance. Specific antibodies may be reacting with neuronal antigens causing CNS disorders in such patients. ${ }^{18}$

In 2001, Al-Rajeh et $\mathrm{al}^{19}$ reported the prevalence of active epilepsy as 6.54 per 1000 , which is comparable to studies from both developed and developing countries. A national Saudi cross-sectional study carried out by Al-Herbish et $\mathrm{al}^{20}$ between 2001 and 2007 to determine the prevalence of IDDM among Saudi children and 
adolescents aged 0-19 years; suggested a prevalence rate of 109.5 per 100,000 .

In 32 studies from different parts of the world, the average prevalence rate was estimated to be 5.16 per $1000 .^{20}$

Children with IDDM have an increased risk of seizures at glycemic extremes-both hypoglycemia and hyperglycemia. ${ }^{17}$ Hypoglycemia used to be a common problem in IDDM patients, and the relationship between hypoglycemia and epilepsy is complicated. Diabetes etiology and metabolic abnormalities of hypo and hyperglycemias may damage the human CNS and trigger subsequent seizures. ${ }^{21}$ Therefore, GAD65 antibodies should be evaluated to help determine this association.

A large body of evidence found that anti-GAD65 antibodies are present in $80 \%$ of IDDM patients. Furthermore, $6 \%$ of epileptic patients have increased GAD65 antibodies. The severity of epilepsy is increased by increasing the serum level of GAD65 antibodies. ${ }^{5}$ However, the etiological association between IDDM, epilepsy, and high levels of GAD65 antibodies remains unclear.

Given the risk of neurological complications associated with both epilepsy and IDDM-as well as the medical and social costs-we need to further investigate the link between both diseases. For these complicated relations between anti-GAD65 antibodies and epilepsy in IDDM children, we tried to determine the prevalence of epilepsy in children with IDDM aged $<15$ years old as well as to study the relationship between epilepsy and elevated anti-GAD65 antibodies.

This study established a significant relation between the presence of IDDM and epilepsy in children. We further confirmed a relationship between pediatric epilepsy and IDDM in the presence of GAD65 antibodies. While our aim was not to study other variants, we found a chance to comment on other observations. We found statistical significance between having epilepsy and a family history of febrile seizures. We found no statistically significant relation between the presence of epilepsy in IDDM in children and factors such as gender, age of onset of diabetes, family history of diabetes, or epilepsy. After effortful searching, we believe that this study might be the first in Saudi Arabia to address the higher prevalence of epilepsy in pediatric IDDM population.

We observed a deficiency in research addressing the link between epilepsy and the presence of anti-GAD65 antibodies in IDDM.

Limitations. Our study is limited by the absence of quantitative measurement of GAD65 antibodies. The latter could have shown possible relation between the level of GAD65 antibodies and the severity of epileptic seizures. Also the study ran in one center, despite being one of the biggest centers, it could have been of more power if it was carried out at multicenter level.

In conclusion, there is a consistent relation between IDDM and epilepsy in children and between epilepsy and positive GAD65 antibodies in case a child is having IDDM.

\section{References}

1. Marcovecchio ML, Petrosino MI, Chiarelli F. Diabetes and epilepsy in children and adolescents. Current Diabetes Reports 2015; 15: 21.

2. Aaberg KM, Gunnes N, Bakken IJ, Lund Søraas C, Berntsen A, Magnus P, Lossius MI, Stoltenberg C, Chin R, Surén P. Incidence and Prevalence of Childhood Epilepsy: A Nationwide Cohort Study. Pediatrics 2017; 139: e20163908.

3. Marcovecchio ML, Petrosino MI, Chiarelli F. Diabetes and epilepsy in children and adolescents. Curr Diab Rep 2015;15: 21.

4. Chou IC, Wang CH, Lin WD, Tsai FJ, Lin CC, Kao CH. Risk of epilepsy in type 1 diabetes mellitus: a population-based cohort study. Diabetologia 2016; 59: 1196-1203.

5. Keezer MR, Novy J, Sander JW. Type 1 diabetes mellitus in people with pharmacoresistant epilepsy: Prevalence and clinical characteristics. Epilepsy Res 2015; 115: 55-57.

6. Fisher RS, van Emde Boas W, Blume W, Elger C, Genton P, Lee $\mathrm{P}$, et al. Epileptic seizures and epilepsy: definitions proposed by the International League against Epilepsy (ILAE) and the International Bureau for Epilepsy (IBE). Epilepsia 2005; 46: 470-472.

7. Bartlett MS. Properties of sufficiency and statistical tests. Proc. Roy Soc Lond Ser 1937; 160: 268-282.

8. Lavrakas P. Encyclopedia of survey research methods. London (UK): SAGE; Statistics.laerd.com. 2019. Available at: https:// statistics.laerd.com/spss-tutorials/independent-t-test-usingspss-statistics.php

9. Tsirogianni A, Pipi E, Soufleros K. Specificity of islet cell autoantibodies and coexistence with other organ specific autoantibodies in type 1 diabetes mellitus. Autoimmun Rev 2009; 8: 687-691.

10. Abdullah MA. Epidemiology of type I diabetes mellitus among Arab children. Saudi Med J 2005; 26: 911-917.

11. Erdö SL, Wolff JR. gamma-Aminobutyric Acid Outside the Mammalian Brain. J Neurochem 1990; 54: 363-372.

12. Erlander MG, Tobin AJ. The structural and functional heterogeneity of glutamic acid decarboxylase: A review. Neurochemical Research 1991; 16: 215-226.

13. Kulaylat NA, Narchi H. A twelve-year study of the incidence of childhood type 1 diabetes mellitus in the Eastern Province of Saudi Arabia. J Pediatr Endocrinol Metab 2000; 13: 135-140.

14 Caietta E, Halbert C, Lépine A, Khammar A, Cano A, Gavaret $\mathrm{M}$, et al. [Association of type 1 diabetes mellitus and epilepsy in children. A cohort of 10 cases]. Arch Pediatr 2011; 19: 9-16. French 
15. Farsani SF, Souverein PC, van der Vorst MM, Knibbe CA, de Boer A, MantelTeeuwisse AK. Chronic comorbidities in children with type 1 diabetes: A population-based cohort study. Arch Dis Child 2015; 100: 763-768.

16. O'connell MA, Harvey AS, Mackay MT, Cameron FJ. Does epilepsy occur more frequently in children with Type 1 diabetes? J Paediatr Child Health 2008; 44: 586-589.

17. Falip M, Miró J, Carreño M, Jaraba S, Becerra JL, Cayuela N. Hypoglycemic seizures and epilepsy in type I diabetes mellitus. J Neurol Sci 2014; 346: 307-309.

18. Bien CG, Scheffer IE. Autoantibodies and epilepsy. Epilepsia 2011; 52: 18-22.
19. Al Rajeh S, Awada A, Bademosi O, Ogunniyi A. Seizure. The prevalence of epilepsy and other seizure disorders in an Arab population: a community-based study. Seizure 2001; 10: 410-414.

20. Al-Herbish AS, El-Mouzan MI, Al-Salloum AA, Al-Qurachi MM, Al-Omar AA. Prevalence of type 1 diabetes mellitus in Saudi Arabian children and adolescents. Saudi Med J 2008; 29: $1285-1288$.

21. Verrotti A, Scaparrotta A, Olivieri C, Chiarelli F. Mechanisms in endocrinology: Seizures and type 1 diabetes mellitus: Current state of knowledge. Eur J Endocrinol 2012; 167: 749-758.

\section{Ethical Consent}

All manuscripts reporting the results of experimental investigations involving human subjects should include a statement confirming that informed consent was obtained from each subject or subject's guardian, after receiving approval of the experimental protocol by a local human ethics committee, or institutional review board. When reporting experiments on animals, authors should indicate whether the institutional and national guide for the care and use of laboratory animals was followed. 